\title{
Biogeography and Microscale Diversity Shape the Biosynthetic Potential of Fungus- growing Ant-associated Pseudonocardia
}

\author{
Bradon R. McDonald ${ }^{1}$, Marc G. Chevrette ${ }^{1,2}$, Jonathan L. Klassen ${ }^{3}$, Heidi A. Horn ${ }^{1}$, Eric J. Caldera ${ }^{1}$, \\ Evelyn Wendt-Pienkowski ${ }^{1}$, Matias J. Cafaro ${ }^{4}$, Antonio C. Ruzzini ${ }^{5}$, Ethan B. Van Arnam ${ }^{5}$, George M. \\ Weinstock ${ }^{6}$, Nicole M. Gerardo ${ }^{7}$, Michael Poulsen ${ }^{8}$, Garret Suen ${ }^{1}$, Jon Clardy ${ }^{5}$, and Cameron R. \\ Currie $^{1}$
}

1 Dept. of Bacteriology, University of Wisconsin-Madison

2 Dept. of Genetics, University of Wisconsin-Madison

3 Dept. of Molecular and Cell Biology, University of Connecticut

4 Dept. of Biology, University of Puerto Rico Mayaguez

5 Dept. of Biological Chemistry and Molecular Pharmacology Harvard Medical School

6 The Jackson Laboratory for Genomic Medicine, Farmington CT

7 Dept. of Biology, Emory University

8 Dept. of Biology, University of Copenhagen

Corresponding Author: Cameron R. Currie (currie@bact.wisc.edu)

\begin{abstract}
The geographic and phylogenetic scale of ecologically relevant microbial diversity is still poorly understood. Using a model mutualism, fungus-growing ants and their defensive bacterial associate Pseudonocardia, we analyzed genetic diversity and biosynthetic potential in 46 strains isolated from ant colonies in a $20 \mathrm{~km}$ transect near Barro Colorado Island in Panama. Despite an average pairwise core genome similarity of greater than $99 \%$, population genomic analysis revealed several distinct bacterial populations matching ant host geographic distribution. We identified both genetic diversity signatures and divergent genes distinct to each lineage. We also identify natural product biosynthesis clusters specific to isolation locations. These geographic patterns were observable despite the populations living in close proximity to each other and provides evidence of ongoing genetic exchange. Our results add to the growing body of literature suggesting that variation in traits of interest can be found at extremely fine phylogenetic scales.
\end{abstract}




\section{Introduction}

The microbial world encompasses a vast amount of phylogenetic, genomic, and ecological diversity (1). However, linking sequence-based metrics of diversity to differences in ecological characteristics remains difficult (2). One major challenge is that a given taxonomic level is generally defined much more broadly in microbes than in eukaryotes. For example, even "closely-related" bacterial groups such as Salmonella and E. coli are estimated to have diverged approximately 100 million years ago (3). In the genus Streptomyces, studies of strains with nearly identical 16S rRNA gene sequences display both diverse antibiotic resistance and resource use phenotypes (4), and retain spatial distributions influenced by glacial movement in the last ice age (5). Additionally, predicting and investigating ecologically relevant phenotypes in microbes is extremely challenging. For example, Vibrio cyclotrophicus strains isolated from different size oceanic organic particles displayed divergence in only a small number of specific genes (6). Even with these data as a guide, significant experimental examination was required to reveal subtle but ecologically significant phenotypic differences that helped explain their physical distribution (7).

Although the environmental forces driving the distribution of fine-scale microbial diversity are poorly understood for most taxa, those that are associated with extreme environmental conditions (8) or with eukaryotic hosts (9) can be used to address biogeographical and population-scale ecological questions more readily. Lineages of bacteria from the actinobacterial genus Pseudonocardia that form a defensive mutualism with many fungus-growing ant species $(10,11)$ provide a useful model system to do this. These filamentous spore forming bacteria grow on the external surface of the ants, where they produce natural products that inhibit the growth of Escovopsis, a co-evolved pathogen of the ant's fungus garden (12). Transmission occurs from one ant to another within the first hours of adult life (13). Queens generally carry the bacteria with them when forming a new colony, but there is phylogenetic evidence of host switches over evolutionary time and potential acquisitions of new symbionts from the environment (14). These bacteria have also become a source of novel small molecules $(15,16)$.

We hypothesized that the genomic diversity of ant-associated Pseudonocardia and their natural product repertoire would vary with host biogeography even over relatively small distances. We investigated this using genomes of Pseudonocardia isolated from Apterostigma ants within a 20 $\mathrm{km}$ transect on and around Barro Colorado Island $(\mathrm{BCl})$ in Panama which formed a single clade in a recent multilocus phylogeny (17). This island was formed from a hilltop that became isolated due to the influx of water that created the Panama Canal. Using a combination of comparative genomic and population genetics, we investigated micro-scale diversity of genome content, genetic exchange, and metabolic potential across kilometer-scale geographic space. 


\section{Results}
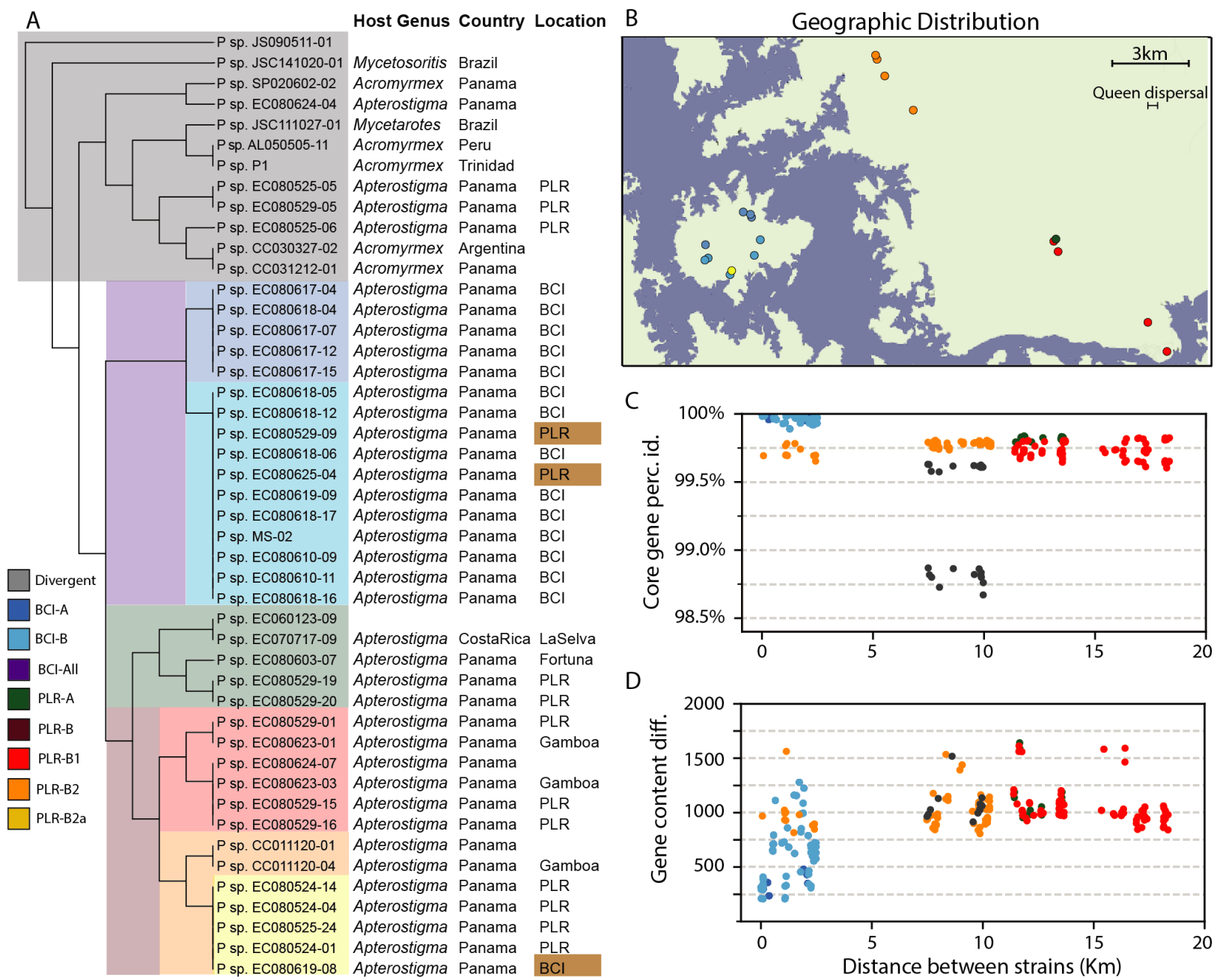

Figure 1: SNP-based clustering of ant-associated Pseudonocardia. A. fineSTRUCTURE clustering of Pseudonocardia strains using 270,000 dimorphic SNP positions. Ant host and isolation location are shown to the right. Lineage colors are used throughout the study. Strains whose local isolation location conflicts with others in their cluster are label in tan. B. Isolation locations based on available GPS data are shown for Pseudonocardia strains in the area around Barro Colorado Island. Strains are colored by fineSTRUCTURE population. C. Core gene percent identity between $\mathrm{BCl}$ cluster strains (purple lineage in $\mathrm{A}$ ) and all strains with GPS coordinates. Points are colored by strain population. D. Raw number of gene content differences between $\mathrm{BCl}$ cluster strains and all others with GPS coordinates.

The 46 Pseudonocardia genomes had a median size of $6.68 \mathrm{mb}$, with a median GC content of $73.42 \%$ and 7,253 open reading frames (ORFs) and inferred RNA coding regions. The number of ORFs is likely inflated by the poor assembly quality of illumina sequenced genomes, as the median number of coding regions in high quality genomes (fewer than 10 contigs) is 5935 (Supp Table 1). These genomes had extremely high sequence similarity, with on average $99.4 \%$ nucleotide identity in core genes (Figure 1). We identified 280,007 dimorphic SNP positions by mapping all genomes in the clade to the complete genome of Pseudonocardia sp. EC08062504, also associated with Apterostigma. Putative population assignments using the SNP data matched the isolation locations for most strains, with two groups of $\mathrm{BCl}$ isolated strains, $\mathrm{BCl}-\mathrm{A}$ 
(light blue) and $\mathrm{BCl}-\mathrm{B}$ (dark blue), and a number of strains from the mainland around Pipeline Road (PLR), labeled PLR-A (green), PLR-B1 (red), and PLR-B2 (orange) (Figure 1a,b).

The SNP-based clusters of $\mathrm{BCl}$ strains largely matched their geographic distribution on the island, with the less diverse $\mathrm{BCl}-\mathrm{A}$ subpopulation occupying the northern and western part of the island. PLR strains also clustered by geographic distribution, with PLR-B2 being in the northern part of our sampling area, while the sympatric populations PLR-A and B1 were isolated $7.6 \mathrm{~km}$ further south. Finally, three strains of PLR Pseudonocardia grouped with Pseudonocardia isolated from other fungus-growing ant species or from $A$. dentigerum colonies from other countries (grey). Sampling of these lineages is more limited in our dataset, with many strain clusters consisting of only one or two genomes. There were three strains whose cluster assignment conflicted with their isolation location: two mainland-isolated strains clustered in BCl-B (Pseudonocardia sp. EC080625-04 and EC080529-09) and a single BCl-isolated strain (EC080619-08) clustered with PLR-B2 (Figure 1a).

The number of polymorphic sites between genomes in reference-aligned regions ranged from 300 to more than 200,000 (Supp Fig 1). Overall, core gene percent identity between isolates from the $\mathrm{BCl}$ populations and mainland genomes matched their geographic locations (Figure 1c, Supp Fig 2a). Except for the isolate that grouped with the mainland strains, $\mathrm{BCl}$ strains had very high sequence similarity with other isolates from the island, and lower core gene similarity to mainland strains. Nearly all strains from the area around $\mathrm{BCl}$ shared core gene percent identities above $99.5 \%$, except for three strains with a percent identity to the $\mathrm{BCl}$ strains of $98.8 \%$.

Genome content diversity also followed a geographical pattern, with $\mathrm{BCl}$ strains generally being similar in genome content (Figure 1d, Supp Fig 2b). Gene content of strains from the BCI populations differed from mainland strains by around $15 \%$. Between $\mathrm{BCl}$ strains, gene content differences range from 188 to 2,063 genes. The pan-genome size of these closely-related Pseudonocardia strains was relatively small, with 6,617 gene families present in at least one genome (Supp Fig 3). The core genome is approximately 3,200 gene families, which represents about half of the total genes found in most Pseudonocardia genomes in our dataset.

Analysis of contigs mapping to the reference genome provided insight into the source of gene content variation between these genomes. The vast majority of contigs either mapped to the reference across nearly their entire length, or failed to map almost entirely. Cases of only part of a contig mapping to the reference were very low, at only $3.5 \%$ of contigs with more than $10 \%$ and less than $90 \%$ mapping to the reference. Further, non-mapping contigs had a lower GC content than mapping contigs, at $72 \%$ versus $74 \%$ respectively (t-test statistic -19.7 , $p$-value 2.82E-85). Many pathways associated with secondary metabolism were over-represented among genes that did not map to the reference genome, along with genes for the degradation of 
xenobiotics and a number of amino acids, and transposon/phage genes. Genes involved in many core biological functions and metabolic pathways were under-represented among genes that did not map to the reference, as expected for conserved functions.

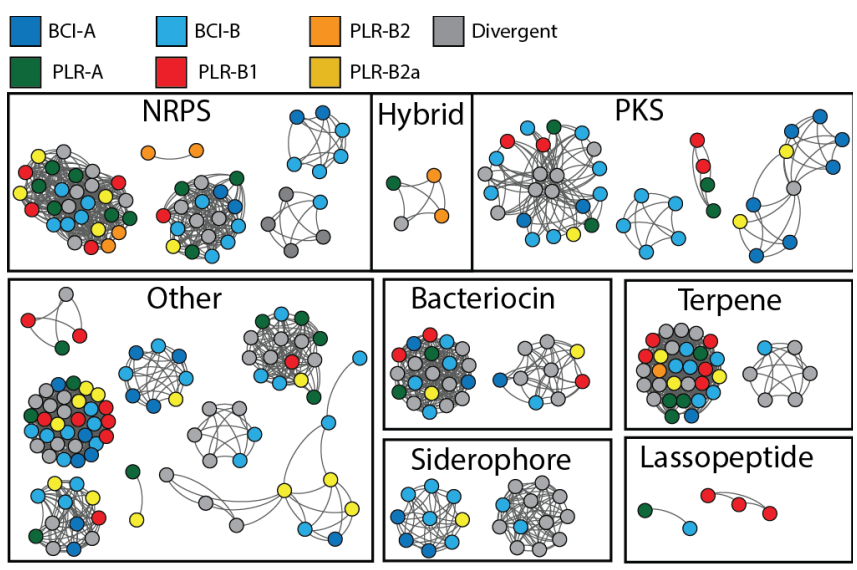

Figure 2: Recurrent natural product biosynthetic gene clusters. Each node represents a contiguous set of genes that are part of a natural product biosynthesis cluster. Edges represent at least $80 \%$ nucleotide sequence identity and $50 \%$ coverage between gene sets and are unweighted. Nodes are colored by their population of origin (see Figure 1).

Since the production of antimicrobial compounds is thought to be the primary ecological role of ant mutualist Pseudonocardia, we investigated the diversity and distribution of natural product biosynthetic gene cluster families (BGCs) among the sampled Pseudonocardia populations (Fig 2). We identified $27 \mathrm{BCG}$ families, 7 of which were only common in the southern $\mathrm{BCl}$ population $\mathrm{BCl}-\mathrm{B}$. Four additional BGCs were significantly enriched among $\mathrm{BCl}$ populations, including a siderophore and two type-II polyketide BCGs. Mainland-specific BGCs included a predicted lassopeptide found only in isolates from the southern part of the mainland area, and a nonribosomal peptide synthetase found only in the northern mainland area. Multiple BGCs, including an ectoine, an oligosaccharide, a terpene, and a nonribosomal peptide synthetase were present within mostly distant populations while being underrepresented or absent in the $\mathrm{PLR}$ and $\mathrm{BCl}$ populations.

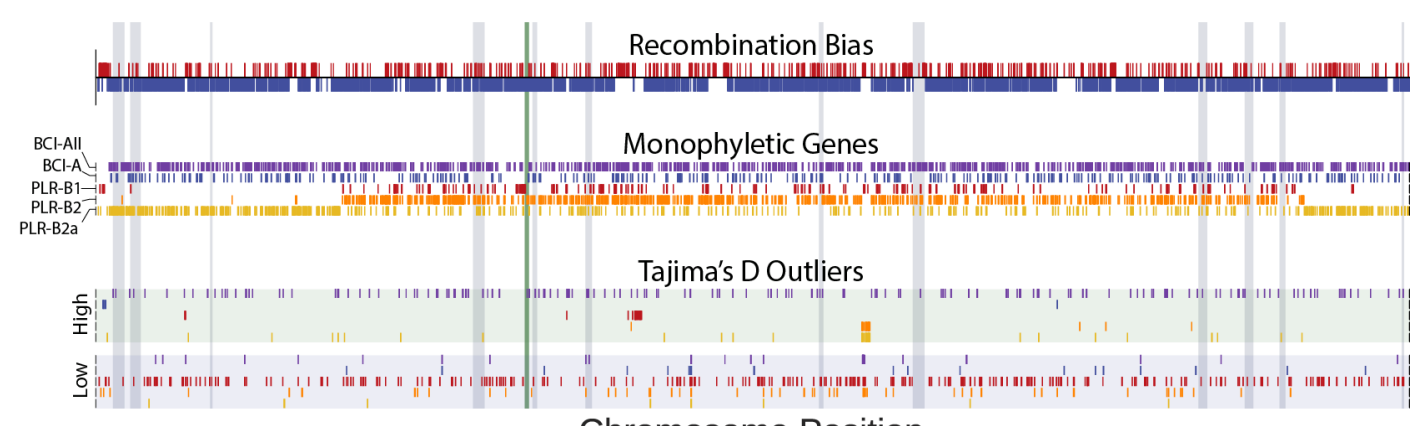

Chromosome Position

Figure 3: Chromosome map of recombination events and divergent genes. All genomic contigs were mapped to the complete genome of Pseudonocardia EC080625-04. Grey regions indicate natural product biosynthesis clusters. The vertical green bar indicates the inferred origin of replication. Populations are colored as in Figure 1. Recombination track shows chromosome regions enriched (red) or depleted (blue) of recombination events. Monophyletic Genes track shows the chromosomal location of genes whose phylogenies form monophyletic groups for each population. Tajima's D Outliers track shows the location of genes whose Tajima's D values deviate by more than 2 standard deviations from the mean of each population. 
Recombination events were non-randomly distributed across the chromosome, with some regions unaffected by recombination events and others affected by as many as 50 events. Nearly all large blocks of high recombination density overlap with transposons and hypothetical proteins. The amount of recombination detected in each strain varied considerably, from $0.1 \%$ to $14.1 \%$ with a median of $5.9 \%$ (Figure 3A). Genes that formed monophyletic clades within each genome cluster, and therefore were not significantly affected by recombination between populations, were generally distributed across the chromosome in $\mathrm{BCl}$ populations (Figure $3 \mathrm{~b}$ ). However, in the PLR lineages there was a pronounced bias. The 248 and 784 monophyletic genes in the red and orange lineages, respectively, were found almost exclusively in the middle portion of the reference genome chromosome, surrounding the origin of replication. This bias was reversed among the 581 monophyletic genes in PLR-B2a (gold). In this lineage, the outer portions of the chromosome were enriched in monophyletic genes.

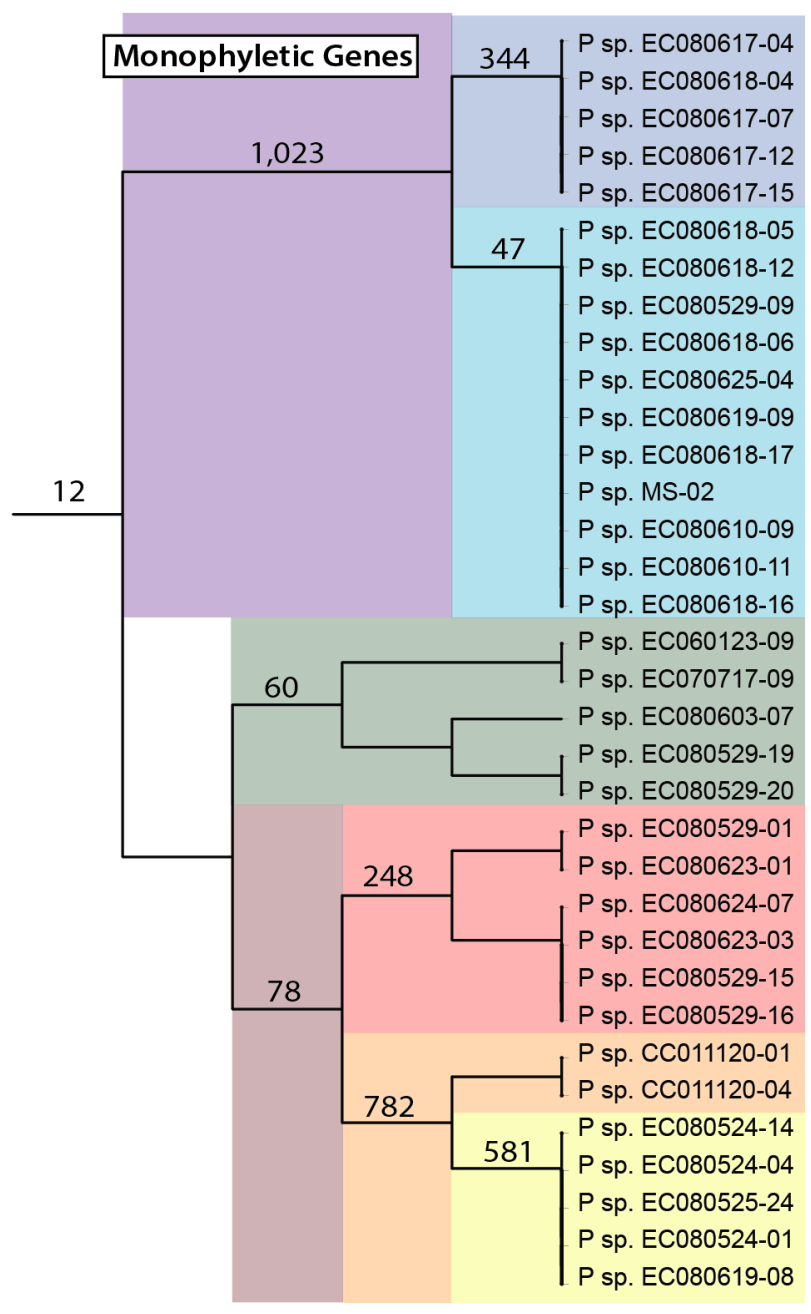

Figure 4: Pipeline road region populationspecific gene divergence. The number of genes that exhibit monophyly for the strains in each lineage containing at least 5 strains are shown on the appropriate branch. 
The ancestral node shared by $\mathrm{BCl}-\mathrm{A}$ and $\mathrm{B}$ (purple) had the highest number of monophyletic genes, at 1,023, while $\mathrm{BCl}-\mathrm{A}$ and $\mathrm{BCl}-\mathrm{B}$ were distinguished by 344 and 43 genes, respectively (Figure 4). Functional categories enriched among $\mathrm{BCl}$ monophyletic gene trees include xenobiotics degradation (odds ratio 1.69), tryptophan metabolism (odds ratio 2.18), ABC transporters (odds ratio 1.67), and nucleotide excision repair (odds ratio 4.64). KEGG gene categories enriched among the monophyletic genes in PLR-B2 (red) include secondary metabolism (odds ratio 1.82) and aminobenzoate degradation (odds ratio 2.21).

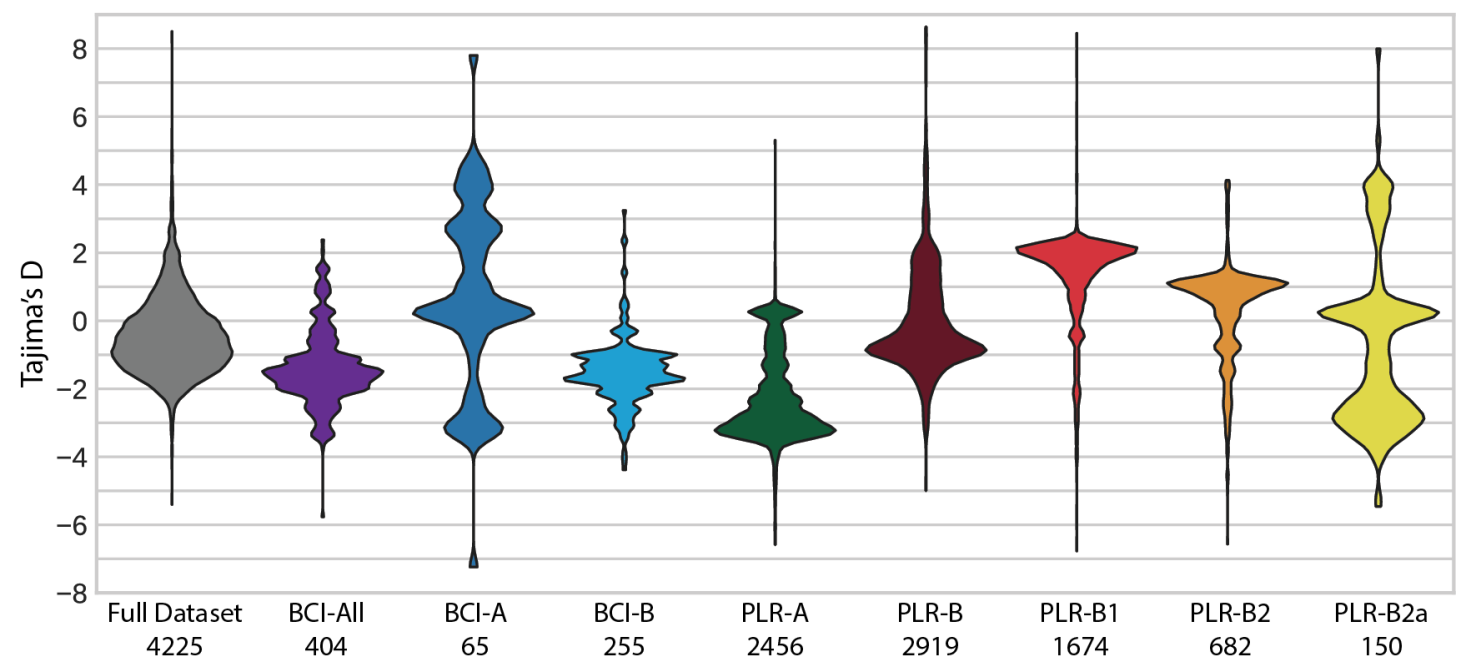

Figure 5: Genetic diversity of conserved genes. Violin plot of the distribution of Tajima's D values for the specified strain group in genes containing at least $1 \%$ polymorphic sites. The number below each strain group name indicates the number of genes analyzed.

We calculated Tajima's D (18) values (TD) for all genes with greater than $1 \%$ polymorphic sites in various strain clusters (Figure 5) to investigate gene-level sequence diversity. The full populationscale dataset had a mean TD value of -0.444 across 4,225 genes. The $\mathrm{BCl}$ strain groups had much lower genetic diversity, with 404 genes containing enough polymorphic sites to calculate TD and a mean value of -1.41 . While the PLR-A cluster also had a low TD value, PLR-B (dark red) had a median TD value of -0.03 , and the PLR-B1, and PLR-B2 had mean TD values of 1.28 and 0.28 , respectively.

We also investigated genes with TD values that deviate from the mean by more than 2 standard deviations in each population, and in all genomes combined. In most individual populations these genes were spread across the genome, except for those in PLR-B (dark red) that cluster near the origin, and a cluster of genes with high TD in PLR-B2 (orange) and B2A (gold) that appear to be part of a mobile element (Figure 3). Across each individual population, genes containing the PFAM (19) domain of unknown function DUF222 were significantly enriched among genes with unusually low TD values (Fisher's exact test, odds ratio 50.02, p value 1.2E-9). Eleven genes in EC080625-04 contain this domain, eight of which are in the lowest $5 \%$ of TD values in at least one population and seven of which are in the lowest $5 \%$ in at least three populations. Two other 
PFAM domains were enriched in the low TD genes, one of which is transposase related (DDE_Tnp) and another a repeat domain of unknown function (RCC1_2).

When the full set of genomes were analyzed as a single dataset, a number of gene clusters showed high TD values. These included several transporters and signaling proteins, along with a cluster of genes involved in exopolysaccharide and cell envelope biosynthesis. Genes with low TD values were more scattered and included a putative prophage and several transposases in addition to the aforementioned DUF222 domains. There were several clusters of low TD genes, including a number of genes in a type-VI secretion system. A number of core genes also show abnormally low TD, including FtsQ and cytochrome $\mathrm{C}$ oxidase subunit I. When the two $\mathrm{BCI}$ populations were analyzed together, low TD genes included a large number of hypothetical proteins along with two secretion-system associated proteins, one type IV VirD4 family and one type VII EccB family. High TD genes included an MT0933-like antitoxin protein along with a range of hypothetical proteins.

\section{Discussion}

Population genomic analysis of Pseudonocardia associated with fungus-growing ants provides a significant contribution to the growing body of literature demonstrating that extremely closelyrelated bacteria, well within standard OTU definitions, can exhibit both gene content diversity and distinct signatures of population dynamics (4-7). Geography plays a clear role in structuring the genome sequence similarity and gene content diversity of Pseudonocardia, with $\mathrm{BCl}$ isolates being highly similar and forming a single lineage that is distinct from most mainland strains. The only $\mathrm{BCl}$-isolated strains that do not share very high sequence similarity to the others instead share high identity with some mainland isolates, suggesting either continuing migration of ant hosts between the island and mainland or sustained coexistence of two lineages since the formation of the island. Similarly, two mainland strains share high sequence similarity with the island strains.

The distribution of natural product BGC families also follows isolation location at this fine geographic scale, as a number of BGC families were found only in $\mathrm{BCl}$ strains. This may be due to geographic diversity of fungus-growing ant pathogens, as dynamic natural product potential is likely important for maintaining effective inhibition of pathogenic fungi. The acquisition of several of these clusters by the recent migrant strain Pseudonocardia sp. EC080619-08 may suggest either rapid acquisition of ecologically relevant genes after host migration, and/or strong selection from pathogens or other environmental conditions prevent the colonization of $\mathrm{BCl}$ by ant hosts whose Pseudonocardia lack the ability to produce particular small molecules. More samples from both mainland and $\mathrm{BCl}$ populations would help address this question by providing a more comprehensive view of BGC family conservation and diversity across locations. Studies on migration and survival of new Apterostigma ant colonies would also shed light on the dynamics of host dispersal and survival in new geographic areas. 
The large number of monophyletic gene trees that separate the $\mathrm{BCI}$ lineage from the mainland lineage provide strong support for genetic isolation of many loci, despite ongoing recombination. This observation is particularly important when investigating evolutionary independence between closely related bacterial lineages. As homologous recombination occurs in relatively small, nonrandom stretches of DNA rather than uniformly across the entire chromosome, bacterial populations can be genetically isolated at some loci while recombining at others (6). This partial genetic isolation model is supported by the strongly biased chromosomal distribution of monophyletic genes in the PLR-B lineages, overlapping with the origin of replication. Such a pattern is consistent with divergence of the core genome despite continued genetic exchange in accessory genes primarily located on the chromosomal periphery, similar to physical distribution of accessory genes on the chromosomes of other Actinobacteria such as Streptomyces (20). Reversal of this trend in PLR-B2a may suggest recombination events or divergence of accessory genes unique to the PLR-B2a lineage. This creates an abundance of monophyletic genes in the periphery of the genome in PLR-B2a strains while they remain less distinguishable from other members of the PLR-B2 lineage in more conserved genes.

Our identification of diverse population dynamics and natural product biosynthetic potential, structured by their geography, suggest this lineage of closely related ant-associated Pseudonocardia contains multiple distinct populations. Categorizing these microbes by sequence similarity alone (21) would incorrectly infer that they are biologically equivalent. Thus, understanding the micro-scale processes that generate microbial diversity requires sampling strategies and analyses that enable very fine resolution $(22,23)$. 


\section{Methods}

Genome assembly and annotation. Pseudonocardia strains were isolated from the cuticle of fungus-growing ants (24) and sequenced using either Pacific Biosciences technology at Duke University (EC080625-04) or Illumina at Washington University in St. Louis. PacBio assemblies were performed using HGAP 1.4 (25), while Illumina genomes were assembled using Velvet (26). Protein coding genes for all genomes were predicted de novo using Prodigal v2.60 (27), while ribosomal RNAs were predicted using RFAM (28) hidden Markov models and Infernal 1.1.1 (29). The origin of replication was predicted using OriLoc (30). Protein coding genes were annotated using TIGRFam v15 (31), PFAM v29, KEGG (32, 33), and actNOG (34) hidden Markov models via HMMer 3.1 (35). Natural product biosynthesis clusters were identified in each genome by antiSMASHv3 (36) followed by manual curation of cluster boundaries. Natural product gene clusters were grouped into families by $80 \%$ nucleotide identity via nucmer (37) alignment and 50\% coverage for each segment of a cluster that matched another. Core and pan genome analyses were conducted using actNOG gene family annotation. All pathway and functional enrichment analyses were based on KEGG annotations.

Population clustering. SNP identification was performed by mapping all genome assemblies to the complete genome of EC080625-04 using nucmer. Reference SNP positions that were covered by a contig for every genome were used for clustering using fineSTRUCTURE (38). Multiple runs with varying values of $c$ and estimated population size had little effect on overall strain clustering, except for very high values of $c$ which caused neighboring clusters to merge (i.e. merging clusters $\mathrm{BCl}-\mathrm{A}$ and $\mathrm{BCl}-\mathrm{B}$ into a single cluster).

Recombination analysis. Recombination events were inferred using bratNextGen (39) on the nucmer alignments of all genomes to EC080625-04, for 10 iterations and 100 replicates with a significance cutoff of 0.01 . The alpha value was reported as 2.7326 . Statistically significant deviations from a random distribution of recombination events were found by comparing the number of recombination events that affected a window to a binomial distribution. A random distribution of recombination would result in each window being affected by approximately 4 events. Blocks of the chromosome that displayed high recombination density were defined as sets of 30 consecutive windows of $500 \mathrm{bp}$ each where the median fold enrichment of recombination events was at least 4 fold.

Conserved gene analysis. Tajima's $D$ and monophyletic gene analyses were conducted using genes from EC080625-04 and the matching regions in contigs from other genomes aligned to this reference. Tajima's D was calculated for genes that had at least $1 \%$ polymorphic nucleotide sites within each population. Monophyletic genes were identified by generating nucleotide alignments for all genes in EC-080625-04 using MAFFT v7.221 (40), followed by gene phylogenies generated using FastTree 2.0 (41). KEGG category enrichment for each lineage was determined using Fisher's Exact Test and a Benjamini-Hochberg false discovery rate of $10 \%$.

Data Accessibility. Genome sequences and annotations are available at DOI: 10.5281/zenodo.2560835

Acknowledgments. This project was supported through National Institutes of Health (NIH) U19 Al109673, NIH U19 TW009872, CAREER Award DEB-747002, National Science Foundation (NSF) MCB-0702025, and NSF MCB-0731822. Additional support was provided to MGC through NIH National Research Service Award T32 GM008505. 
bioRxiv preprint doi: https://doi.org/10.1101/545640; this version posted February 10, 2019. The copyright holder for this preprint (which was not certified by peer review) is the author/funder, who has granted bioRxiv a license to display the preprint in perpetuity. It is made available under aCC-BY-ND 4.0 International license.

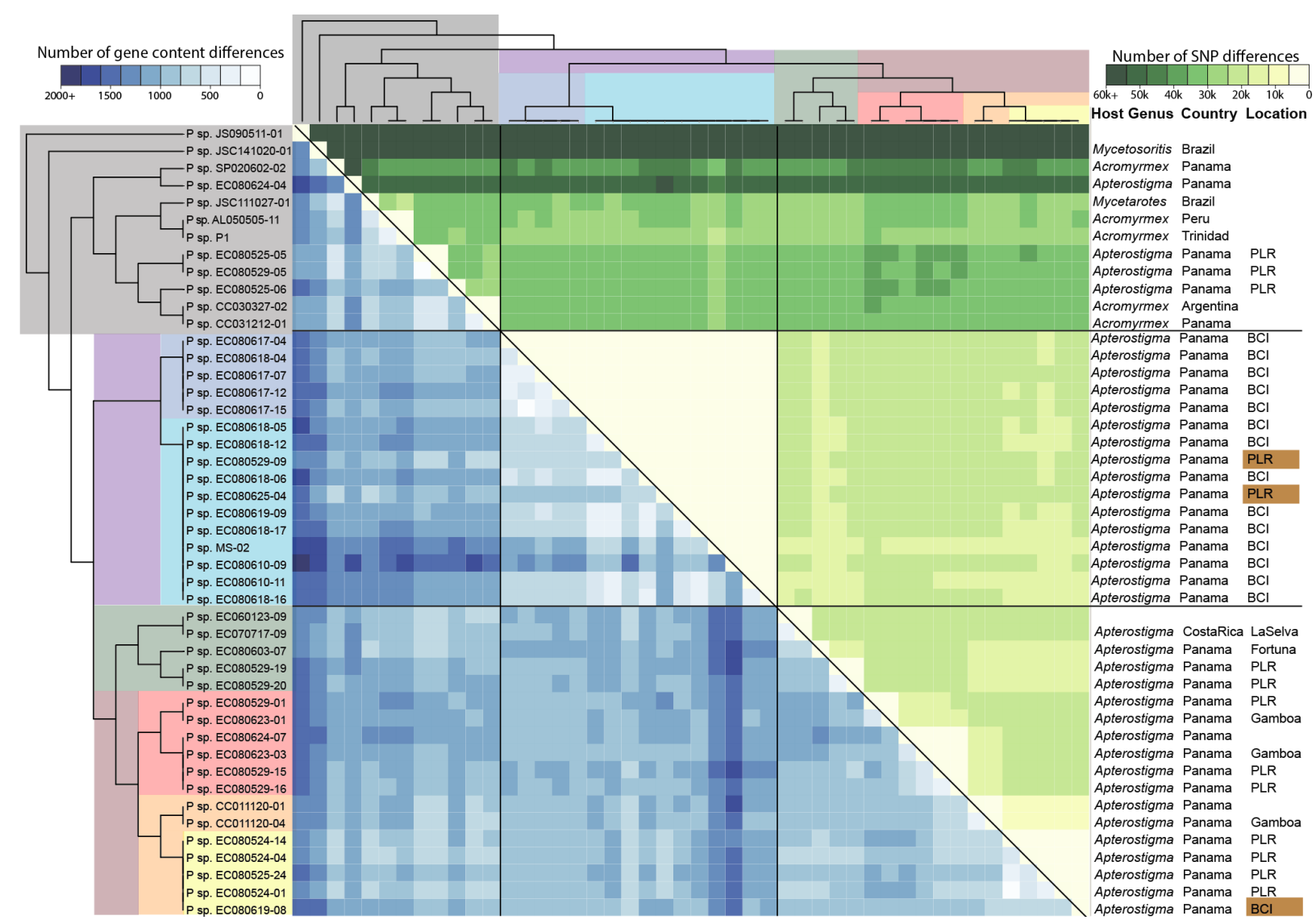

Supplemental Figure 1: Genome content and SNP diversity in ant-associated Pseudonocardia. The number of actNOG gene content differences (blue) or SNP differences (green) between genome pairs. Isolation hosts and locations are given on the right. 
A

EC080618-12

EC080617-04

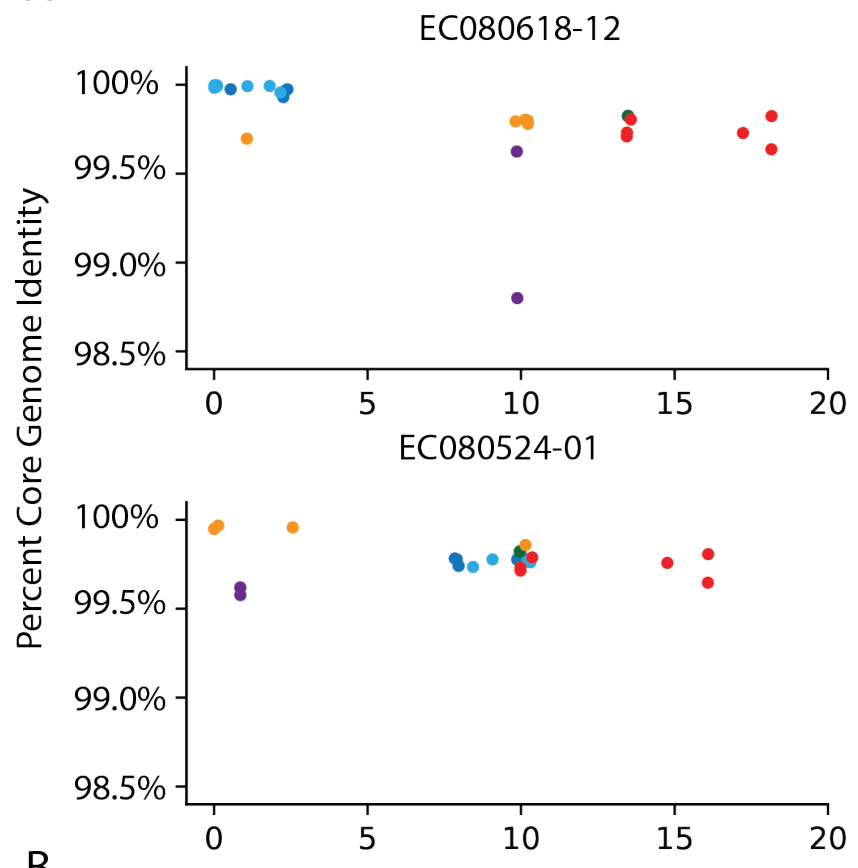

B
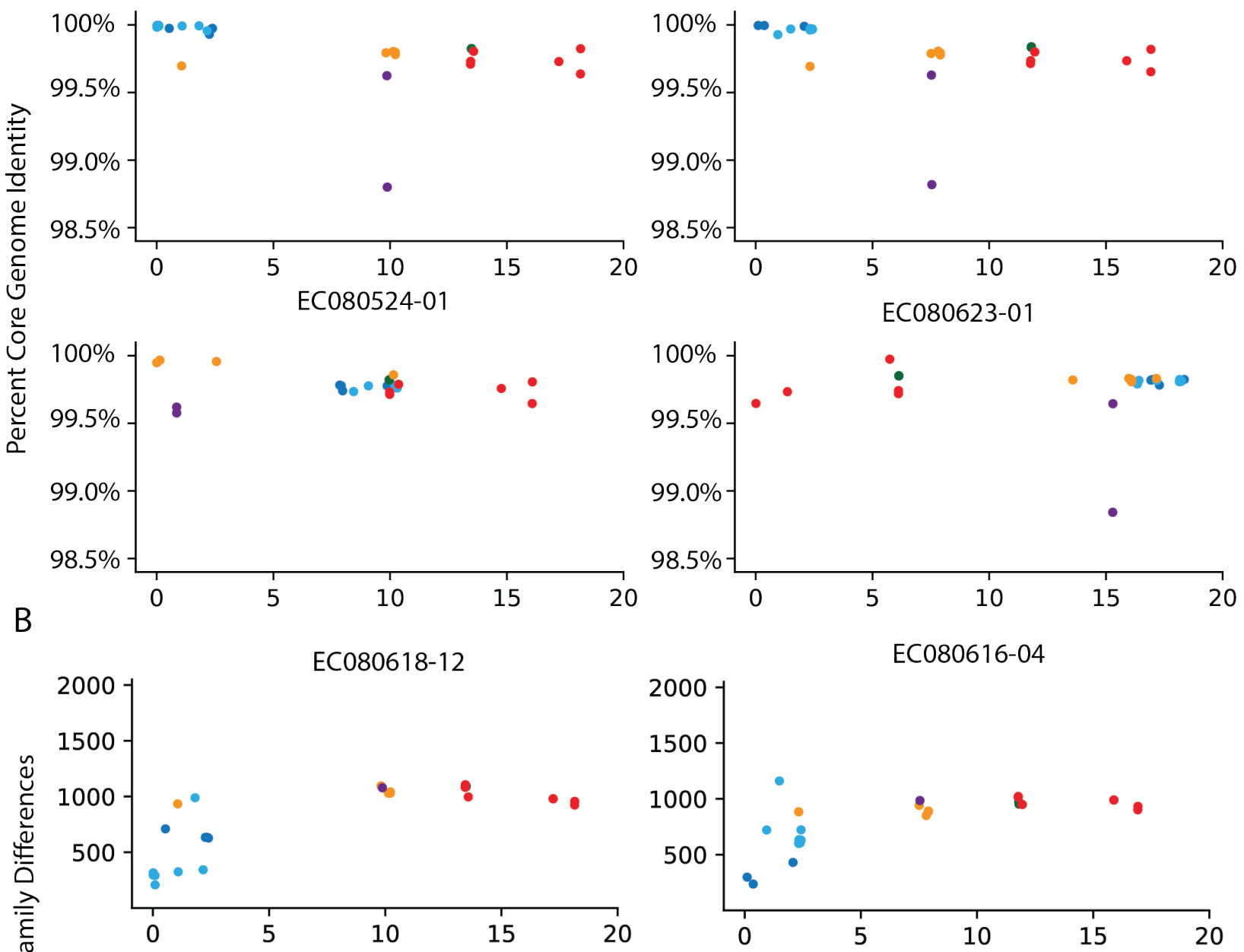

EC080524-01

EC080623-01
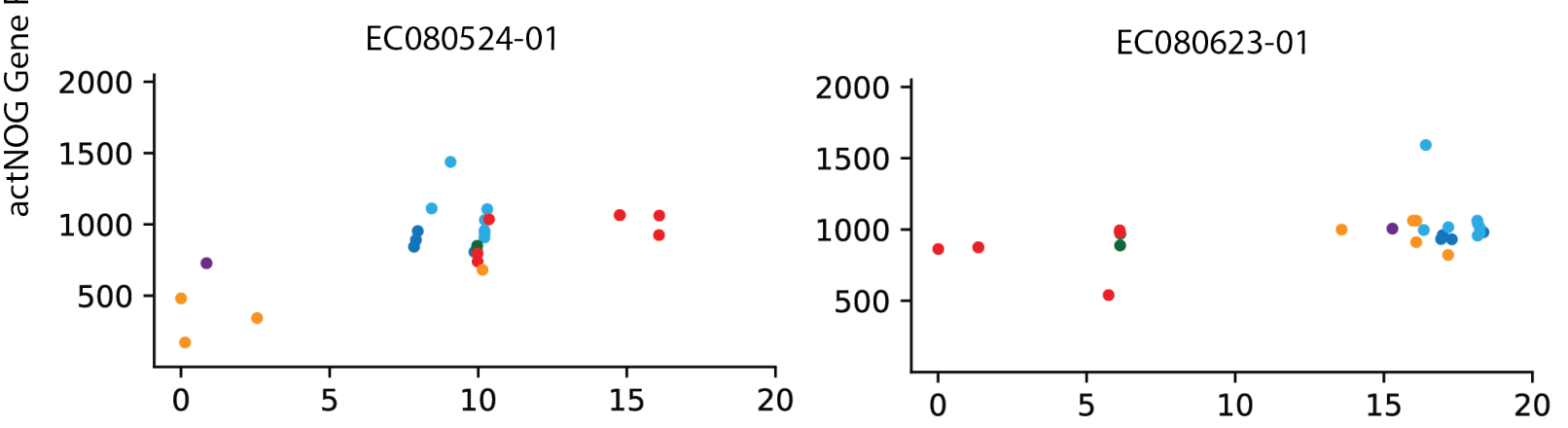

Distance from Reference Strain (Km)

Supplemental Figure 2: Conserved gene divergence and gene content divergence across geographic space A. Core gene percent identity between PacBio assembled reference strains and all strains with GPS coordinates. Points are colored by strain population. Plot titles indicate the strain to which all other strains are compared. B. Raw number of gene content differences between PacBio finished reference strains and all others with GPS coordinates. 


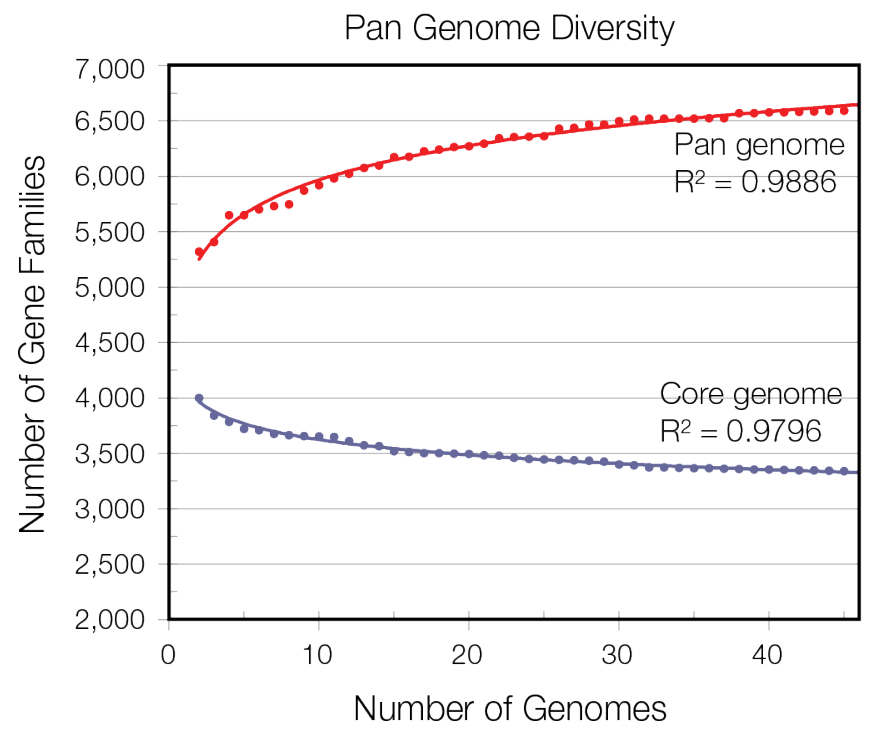

Supplemental Figure 3: Pan-genome and core-genome size in closely related ant-associated

Pseudonocardia. The total number of actNOG gene families present in the genome dataset (red) and the number of actNOG gene families conserved in all genomes (blue), as more genomes are added to the dataset. The pan-genome size fits a logarithmic function, while the core-genome size fits a power-law function. 
Supplementary Table 1. Genome assembly and annotation statistics

\begin{tabular}{|c|c|c|c|c|c|}
\hline Genome & GenomeSize & N50 & TotalContigs & $\mathrm{GC}$ & GeneCount \\
\hline AL050505-11 & 6566921 & 6389648 & 3 & 73.78 & 6046 \\
\hline CC011120-01 & 6369072 & 5458 & 2178 & 73.55 & 7396 \\
\hline CC011120-04 & 6368325 & 6216 & 1884 & 73.56 & 7258 \\
\hline CC030327-02 & 5921325 & 6235 & 1740 & 73.72 & 6891 \\
\hline CC031212-01 & 5889746 & 7200 & 1736 & 73.41 & 6824 \\
\hline EC060123-09 & 6201792 & 8217 & 1463 & 73.74 & 6881 \\
\hline EC070717-09 & 6351919 & 10828 & 1128 & 73.79 & 6753 \\
\hline EC080524-01 & 6189181 & 9548 & 1282 & 73.61 & 6633 \\
\hline EC080524-04 & 6371034 & 13375 & 903 & 73.8 & 6459 \\
\hline EC080524-14 & 6195412 & 10360 & 1087 & 73.73 & 6485 \\
\hline EC080525-05 & 6068818 & 9480 & 1349 & 73.88 & 6586 \\
\hline EC080525-06 & 6836013 & 9998 & 1385 & 73.45 & 7328 \\
\hline EC080525-24 & 6269987 & 6880 & 1629 & 73.54 & 7030 \\
\hline EC080529-01 & 7106933 & 8171 & 1728 & 73.16 & 7892 \\
\hline EC080529-05 & 6103212 & 8937 & 1390 & 73.85 & 6681 \\
\hline EC080529-09 & 6348069 & 6770 & 1714 & 73.56 & 7171 \\
\hline EC080529-15 & 6433317 & 10850 & 1259 & 73.64 & 6870 \\
\hline EC080529-16 & 6427229 & 10977 & 1210 & 73.6 & 6783 \\
\hline EC080529-19 & 6745963 & 8650 & 1626 & 73.4 & 7452 \\
\hline EC080529-20 & 6861062 & 12349 & 1260 & 73.43 & 7207 \\
\hline EC080603-07 & 6481475 & 11157 & 1109 & 73.78 & 6766 \\
\hline EC080610-09 & 7131853 & 6138223 & 3 & 73.34 & 6597 \\
\hline EC080610-11 & 6787135 & 4988 & 2501 & 72.94 & 8225 \\
\hline EC080617-04 & 6932605 & 9808 & 1412 & 73.35 & 7393 \\
\hline EC080617-07 & 6939821 & 11292 & 1271 & 73.29 & 7331 \\
\hline EC080617-12 & 6879092 & 6338 & 2033 & 73.21 & 7935 \\
\hline EC080617-15 & 6789668 & 8761 & 1475 & 73.31 & 7324 \\
\hline EC080618-04 & 6789668 & 8761 & 1475 & 73.31 & 7324 \\
\hline EC080618-05 & 7101947 & 12991 & 1127 & 73.21 & 7359 \\
\hline EC080618-06 & 7107275 & 16463 & 990 & 73.23 & 7295 \\
\hline EC080618-12 & 7021027 & 7448 & 1858 & 73.12 & 7878 \\
\hline EC080618-16 & 6923749 & 5242 & 2468 & 72.99 & 8357 \\
\hline EC080618-17 & 7014064 & 7082 & 1842 & 73.15 & 7863 \\
\hline EC080619-08 & 6766081 & 5881 & 2090 & 73.27 & 7783 \\
\hline EC080619-09 & 7024805 & 14182 & 1039 & 73.28 & 7249 \\
\hline EC080623-01 & 6785441 & 6527 & 1963 & 73.3 & 7798 \\
\hline EC080623-03 & 6881261 & 10164 & 1334 & 73.35 & 7321 \\
\hline EC080624-04 & 6961708 & 7831 & 1962 & 73.21 & 7795 \\
\hline EC080624-07 & 6855742 & 6063 & 2110 & 73.26 & 7919 \\
\hline EC080625-04 & 6135769 & $\mathrm{Na}$ & 1 & 73.84 & 5744 \\
\hline JS090511-01 & 6608484 & 5335 & 2398 & 73.85 & 8033 \\
\hline JSC111027-01 & 6362052 & 6342421 & 2 & 73.95 & 5825 \\
\hline JSC141020-01 & 7303443 & 6658632 & 4 & 73.62 & 6619 \\
\hline MS-02 & 6188306 & 3359 & 3092 & 72.33 & 8189 \\
\hline P1 & 6388771 & 14149 & 875 & 73.25 & 6636 \\
\hline SP020602-02 & 6322523 & $\mathrm{Na}$ & 1 & 74 & 5771 \\
\hline MedianAll & 6677223 & 8849 & 1401 & 73.42 & 7253 \\
\hline MedianHighQuality & 6711593 & 6366034 & 2.5 & 73.81 & 5935 \\
\hline
\end{tabular}




\section{References}

1. Rinke C, Schwientek P, Sczyrba A, Ivanova NN, Anderson IJ, Cheng J-F, Darling AE, Malfatti S, Swan BK, Gies EA, Dodsworth JA, Hedlund BP, Tsiamis G, Sievert SM, Liu W-T, Eisen JA, Hallam SJ, Kyrpides NC, Stepanauskas R, Rubin EM, Hugenholtz P, Woyke T. 2013. Insights into the phylogeny and coding potential of microbial dark matter. Nature 499:431-437.

2. Martiny JBH, Bohannan BJM, Brown JH, Colwell RK, Fuhrman J a, Green JL, Horner-Devine MC, Kane M, Krumins JA, Kuske CR, Morin PJ, Naeem S, Ovreås L, Reysenbach A-L, Smith VH, Staley JT. 2006. Microbial biogeography: putting microorganisms on the map. Nat Rev Microbiol 4:102-112.

3. Lawrence JG, Ochman H. 1998. Molecular archaeology of the Escherichia coli genome. Proc Natl Acad Sci U S A 95:9413-9417.

4. Schlatter DC, Kinkel LL. 2014. Global biogeography of Streptomyces antibiotic inhibition, resistance, and resource use. FEMS Microbiol Ecol 88:386-397.

5. Andam CP, Doroghazi JR, Campbell AN, Kelly PJ, Choudoir MJ, Buckley DH. 2016. A Latitudinal Diversity Gradient in Terrestrial Bacteria of the Genus Streptomyces. MBio 7:1-9.

6. Shapiro BJ, Friedman J, Cordero OX, Preheim SP, Timberlake SC, Szabo G, Polz MF, Alm EJ. 2012. Population Genomics of Early Events in the Ecological Differentiation of Bacteria. Science 336:48-51.

7. Yawata Y, Cordero OX, Menolascina F, Hehemann J-H, Polz MF, Stocker R. 2014. Competitiondispersal tradeoff ecologically differentiates recently speciated marine bacterioplankton populations. Proc Natl Acad Sci U S A 111:5622-7.

8. Cadillo-Quiroz H, Didelot X, Held NL, Herrera A, Darling A, Reno ML, Krause DJ, Whitaker RJ. 2012. Patterns of Gene Flow Define Species of Thermophilic Archaea. PLoS Biol 10:e1001265.

9. Moran NA, McLaughlin HJ, Sorek R. 2009. The dynamics and time scale of ongoing genomic erosion in symbiotic bacteria. Science 323:379-382.

10. Currie CR, Wong B, Stuart AE, Schultz TR, Rehner SA, Mueller UG, Sung GH, Spatafora JW, Straus NA. 2003. Ancient tripartite coevolution in the attine ant-microbe symbiosis. Science 299:386-388.

11. Holmes NA, Innocent TM, Heine D, Al Bassam M, Worsley SF, Trottmann F, Patrick EH, Yu DW, Murrell JC, Schiott M, Wilkinson B, Boomsma JJ, Hutchings MI. 2016. Genome analysis of two Pseudonocardia phylotypes associated with acromyrmex leafcutter ants reveals their biosynthetic potential. Front Microbiol 7:1-16.

12. Currie CR, Scott JA, Summerbell RC, Malloch D. 1999. Fungus-growing ants use antibiotic-producing bacteria to control garden parasites. Nature 398:701.

13. Marsh SE, Poulsen M, Pinto-Tomás A, Currie CR. 2014. Interaction between workers during a short time window is required for bacterial symbiont transmission in acromyrmex leaf-cutting ants. PLoS One 9.

14. Cafaro MJ, Poulsen M, Little AEF, Price SL, Gerardo NM, Wong B, Stuart AE, Larget B, Abbot P, Currie CR. 2011. Specificity in the symbiotic association between fungus-growing ants and protective Pseudonocardia bacteria. Proc Biol Sci 278:1814-22.

15. Van Arnam EB, Ruzzini AC, Sit CS, Horn H, Pinto-Tomás AA, Currie CR, Clardy J. 2016. Selvamicin, an atypical antifungal polyene from two alternative genomic contexts. Proc Natl Acad Sci 113:12940-12945.

16. Van Arnam EB, Ruzzini AC, Sit CS, Currie CR, Clardy J. 2015. A Rebeccamycin Analog Provides Plasmid-Encoded Niche Defense. J Am Chem Soc 137:14272-14274.

17. Caldera EJ, Currie CR. 2012. The Population Structure of Antibiotic-Producing Bacterial Symbionts of Apterostigma dentigerum Ants: Impacts of Coevolution and Multipartite Symbiosis. Am Nat 180:604-617.

18. Tajima F. 1989. Statistical method for testing the neutral mutation hypothesis by DNA polymorphism. Genetics 123:585-595.

19. Finn RD, Bateman A, Clements J, Coggill P, Eberhardt RY, Eddy SR, Heger A, Hetherington K, Holm L, Mistry J, Sonnhammer ELL, Tate J, Punta M. 2014. Pfam: the protein families database. Nucleic Acids Res 42:D222-30.

20. Hopwood DA. 2006. Soil To Genomics: The Streptomyces Chromosome. Annu Rev Genet 40:1-23.

21. Caro-Quintero A, Konstantinidis KT. 2012. Bacterial species may exist, metagenomics reveal. Environ Microbiol 14:347-355.

22. Levy MB. 1992. the Problem of Pattern and Scale in Ecology. Ecology 73:1943-1967.

23. Chave J. 2013. The problem of pattern and scale in ecology: What have we learned in 20 years? Ecol 
Lett 16:4-16.

24. Poulsen M, Cafaro M, Boomsma JJ, Currie CR. 2005. Specificity of the mutualistic association between actinomycete bacteria and two sympatric species of Acromyrmex leaf-cutting ants. Mol Ecol 14:35973604.

25. Chin C-S, Alexander DH, Marks P, Klammer AA, Drake J, Heiner C, Clum A, Copeland A, Huddleston J, Eichler EE, Turner SW, Korlach J. 2013. Nonhybrid, finished microbial genome assemblies from longread SMRT sequencing data. Nat Methods 10:563-9.

26. Zerbino DR, Birney E. 2008. Velvet: Algorithms for de novo short read assembly using de Bruijn graphs. Genome Res 18:821-829.

27. Hyatt D, Chen G-L, Locascio PF, Land ML, Larimer FW, Hauser LJ. 2010. Prodigal: prokaryotic gene recognition and translation initiation site identification. BMC Bioinformatics 11:119.

28. Nawrocki EP, Burge SW, Bateman A, Daub J, Eberhardt RY, Eddy SR, Floden EW, Gardner PP, Jones TA, Tate J, Finn RD. 2015. Rfam 12.0: Updates to the RNA families database. Nucleic Acids Res 43:D130-D137.

29. Nawrocki EP, Eddy SR. 2013. Infernal 1.1: 100-fold faster RNA homology searches. Bioinformatics 29:2933-2935.

30. Frank AC, Lobry JR. 2000. Oriloc: Prediction of replication boundaries in unannotated bacterial chromosomes. Bioinformatics 16:560-561.

31. Haft DH, Selengut JD, Richter RA, Harkins D, Basu MK, Beck E. 2013. TIGRFAMs and genome properties in 2013. Nucleic Acids Res 41:387-395.

32. Moriya Y, Itoh M, Okuda S, Yoshizawa AC, Kanehisa M. 2007. KAAS: An automatic genome annotation and pathway reconstruction server. Nucleic Acids Res 35:182-185.

33. Kanehisa M, Goto S, Sato Y, Kawashima M, Furumichi M, Tanabe M. 2014. Data, information, knowledge and principle: Back to metabolism in KEGG. Nucleic Acids Res 42:199-205.

34. Powell S, Szklarczyk D, Trachana K, Roth A, Kuhn M, Muller J, Arnold R, Rattei T, Letunic I, Doerks T, Jensen LJ, Von Mering C, Bork P. 2012. eggNOG v3.0: Orthologous groups covering 1133 organisms at 41 different taxonomic ranges. Nucleic Acids Res 40:284-289.

35. Eddy SR. 2011. Accelerated profile HMM searches. PLoS Comput Biol 7:e1002195.

36. Weber T, Blin K, Duddela S, Krug D, Kim HU, Bruccoleri R, Lee SY, Fischbach MA, Müller R, Wohlleben W, Breitling R, Takano E, Medema MH. 2015. antiSMASH 3.0-a comprehensive resource for the genome mining of biosynthetic gene clusters. Nucleic Acids Res 43:W237-W243.

37. Kurtz S, Phillippy A, Delcher A, Smoot M, Shumway M, Antonescu C, Salzberg S. 2004. Versatile and open software for comparing large genomes. Genome Biol 5:R12.

38. Lawson DJ, Hellenthal G, Myers S, Falush D. 2012. Inference of population structure using dense haplotype data. PLoS Genet 8:11-17.

39. Marttinen P, Hanage WP, Croucher NJ, Connor TR, Harris SR, Bentley SD, Corander J. 2012. Detection of recombination events in bacterial genomes from large population samples. Nucleic Acids Res 40:e6.

40. Katoh K, Standley DM. 2013. MAFFT multiple sequence alignment software version 7: improvements in performance and usability. Mol Biol Evol 30:772-80.

41. Price MN, Dehal PS, Arkin AP. 2010. FastTree 2 - Approximately maximum-likelihood trees for large alignments. PLoS One 5:e9490. 